\title{
Research on the Power Tracking Control of Permanent Manget Synchronous Wind Turbine with Losses Considered
}

\author{
Liu Fei, Gao Shujing, Ren Minmin \\ School of Information Engineering, Engineering University of PAP, Xi'an, China
}

Email address:

tangsengff@foxmail.com (Liu Fei), 149297683@qq.com (Gao Shujing), violet_212@163.com (Ren Minmin)

\section{To cite this article:}

Liu Fei, Gao Shujing, Ren Minmin. Research on the Power Tracking Control of Permanent Manget Synchronous Wind Turbine with Losses Considered. Science Discovery. Vol. 9, No. 4, 2021, pp. 160-170. doi: 10.11648/j.sd.20210904.16

Received: April 17, 2021; Accepted: May 25, 2021; Published: June 4, 2021

\begin{abstract}
For the permanent magnet synchronous wind generator, a control method based on losses minimization is proposed, in order to solve the problem of the maximum wind energy tracking control, which often neglects the generator losses caused by the deviation between the actual working point and the optimal power point, and leads to the output power loss. Then, considering the loss of generator, the small-signal method is used to establish the transfer functions of the main components, such as the wind turbine, transmission chains, permanent magnet synchronous generator and so on. The zeros and poles of the transfer functions and their related stability are analyzed. The PI controllers of the current loop and power loop are designed, and the calculation method of control parameters is given. The simulation results verify the effectiveness of the proposed method, and show that the maximum wind energy tracking mode based on the loss minimization control (LMC) can reduce the controllable losses of the generator. Finally, compared with the $i_{d}=0$ control and the maximum torque per ampere control (MTPA), the method improves the maximum power tracking performance and increases the power output.
\end{abstract}

Keywords: Wind Turbines, MPPT (Maximum Power Point Tracking), Frequency Domain Analysis, Losses, Linearization, Transfer Functions

\section{考虑永磁同步风力发电机损耗的功率跟踪研究}

刘飞, 高树静, 任敏敏

武警工程大学信息工程学院, 西安, 中国

邮箱

tangsengff@foxmail.com (刘飞)，149297683@qq.com（高树静）, violet_212@163.com（任敏敏）

摘要: 针对忽略发电机损耗情况下, 永磁同步风力发电机进行最大风能跟踪控制时, 实际工作点与理想最佳功率点存 在偏差，造成输出功率损失的问题，提出一种基于最小损耗的控制方式。进而利用小信号法，建立了考虑发电机损耗 情况下包含风力机、传动链、永磁同步风力发电机等主要部件的传递函数, 对系统的零、极点位置及其稳定性进行了 分析, 设计了电流环、功率环PI控制器, 给出了控制参数计算方法。系统仿真验证该方法的有效性, 结果表明基于最 小损耗控制(LMC)的永磁同步风力发电机最大风能跟踪减少了发电机的可控损耗。与 $d$ 轴电流 $\left(i_{d}=0\right)$ 控制和最大转矩电 流比控制(MTPA)进行比较，该方法改善了最大功率跟踪性能，提高了风力发电机输出功率。

关键词: 风力机, 最大功率跟踪, 频域分析, 损耗, 线性化, 传递函数 


\section{1. 引言}

最大功率跟踪作为风力发电机组主要的控制策略, 常 用的方法有叶尖速比法、爬山法、最优转矩控制(OTC) [1,2]、 功率反馈控制、极值搜索控制(ESC)等[3-5]。然而, 风力 发电机损耗对机组动态性能和最大功率点的影响却往往 被忽略。风力发电机的损耗随风速的变化而变化, 忽略发 电机损耗不但导致实际控制中不能准确给定最大参考功 率, 而且推算出的机组工作点也存在明显的偏差[6]。

文献[7]从定量的角度分析了不同风速下功率损耗对 最大功率点的影响。文献[8]研究了损耗对风力发电机控制 有效性的影响, 并分析了最优功率控制中PI控制器的设计。 文献[9]分析了永磁同步风力发电机的效率, 提出一种基于 转速估计的无传感器MPPT控制方案。但是, 二者均未考 虑铁损等因素影响, 未给出具体的控制参数选取方法。文 献[10]采用虚拟信号搜索MTPA控制下的最佳电流角, 避 免了注入激励信号所引起的扰动和额外的电损耗。而最小 损耗控制(LMC)则需综合考虑铜耗和铁耗。文献[11]比较 了MTPA和LMC方法, 说明二者之间存在明显的差异。文 献[12]采用二分查找法搜索最小功率损耗下的 $d$ 轴和 $q$ 轴电 流, 提高了搜索速度。文献[13]和[14]均提出利用多项式

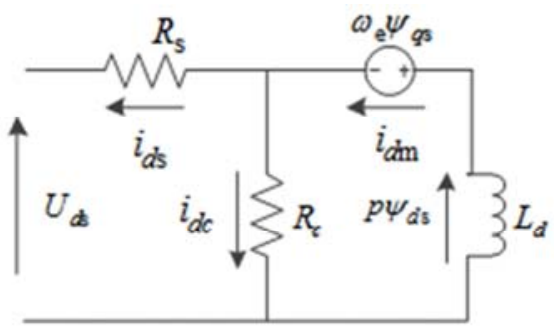

拟合不同工况下永磁同步风力发电机的功率损耗, 从而提 高机组输出功率。但上述方法未对风力发电机动态性能和 控制参数配置做出进一步分析。

本文以永磁同步风力发电机为对象, 在考虑机械损耗、 铜耗、铁耗等损耗的情况下, 建立风力机、发电机和传动链 等主体部件的传递函数模型, 设计了电流环和功率环PI控制 器。采用小信号法分析了不同工况下传递函数零、极点位置 以及机组主要参数对系统动态性能、最大功率点偏移和稳定 性的影响。采用频域法进行分析, 给出控制参数具体的选取 方案。在功率控制回路中, 提出了一种最小功率损耗控制策 略, 在跟踪最大功率点的同时提高了风力发电机运行效率。

\section{2. 电流环分析}

\section{1. 发电机模型}

永磁同步风力发电机的铁耗包括磁滞损耗和浴流损 耗, 可以用等效铁损电阻 $R_{c}$ 表示, $d 、 q$ 轴等效电路如图 1 所示 $[7,15]$ 。

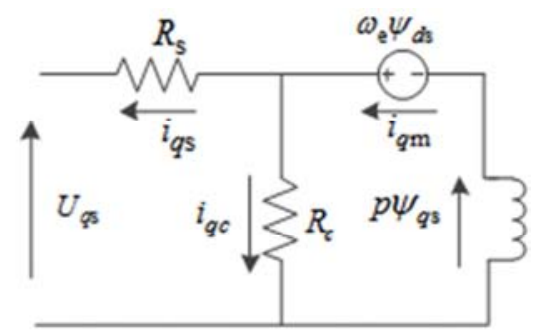

图1 永磁同步电机 $d-q$ 轴等效电路图。

图中, $\mathrm{U}_{d \mathrm{~s}} 、 \mathrm{U}_{q \mathrm{~s}} \longrightarrow d-q$ 旋转坐标系中的定子绕组电压 $d 、 q$ 轴分量; $i_{d \mathrm{~m}} 、 i_{q \mathrm{~m}}$ —气隙磁场产生的电流分量; $i_{d \mathrm{~s}}$ 、 $i_{q \mathrm{~s}} \longrightarrow d 、 q$ 轴定子绕组电流分量; $i_{d c} 、 i_{q c}$ 一等效铁耗电 流 $d 、 q$ 轴分量; $\psi_{d \mathrm{~s}} 、 \psi_{q \mathrm{~s}}$ —定子磁链分量; $R_{\mathrm{s}}$ 一定 子绕组电阻; $\psi_{\mathrm{m}}$ 为永磁磁链; $L_{d} 、 L_{q}$ 一定子绕组 $d$ 轴 和 $q$ 轴电感; $\omega_{\mathrm{e}}$ 为电角速度。结合图 1 可得, 在 $d-q$ 坐标系 下，考虑损耗的永磁同步发电机电压方程为:

$$
\begin{gathered}
U_{d \mathrm{~s}}=-R_{\mathrm{s}} i_{d \mathrm{~s}}-L_{d} \frac{d i_{d \mathrm{~m}}}{d t}+L_{q} i_{q \mathrm{~m}} \omega_{\mathrm{e}} \\
U_{q \mathrm{~s}}=-R_{\mathrm{s}} i_{q \mathrm{~s}}-L_{q} \frac{d i_{q \mathrm{~m}}}{d t}+\left(\psi_{\mathrm{m}}-L_{d} i_{d \mathrm{~m}}\right) \omega_{\mathrm{e}} \\
i_{q \mathrm{~m}}=i_{q \mathrm{~s}}+i_{q c} \\
i_{d \mathrm{~m}}=i_{d \mathrm{~s}}+i_{d c} \\
i_{d c}=\frac{\omega_{\mathrm{e}} L_{q} i_{q \mathrm{~m}}-L_{d} \frac{d i_{d \mathrm{~m}}}{d t}}{R_{c}}
\end{gathered}
$$

$$
i_{q c}=\frac{\left(\psi_{\mathrm{m}}-L_{d} i_{d \mathrm{~m}}\right) \omega_{\mathrm{e}}-L_{q} \frac{d i_{q \mathrm{~m}}}{d t}}{R_{c}}
$$

永磁同步风力发电机输出功率 $P_{\mathrm{g}}$ 可由式(1)-(4)导出, 忽略暂态变量, 输出功率表达式为:

$$
\begin{aligned}
& P_{\mathrm{g}}=\frac{3}{2}\left(U_{d \mathrm{~s}} i_{d \mathrm{~s}}+U_{q \mathrm{~s}} i_{q \mathrm{~s}}\right)=\frac{3}{2}\left[\omega_{\mathrm{e}} \psi_{\mathrm{m}} i_{q \mathrm{~m}}-\omega_{\mathrm{e}}\left(L_{d}-L_{q}\right) i_{d \mathrm{~m}} i_{q \mathrm{~m}}\right. \\
& \left.-R_{\mathrm{s}}\left(i_{d \mathrm{~s}}^{2}+i_{q \mathrm{~s}}^{2}\right)-\omega_{\mathrm{e}} \psi_{\mathrm{m}} i_{q c}+L_{d} i_{d \mathrm{~m}} \omega_{\mathrm{e}} i_{q c}-\omega_{\mathrm{e}} L_{q} i_{q \mathrm{~m}} i_{d c}\right]
\end{aligned}
$$

式(7)分解后, 前 2 项为发电机输入功率, 第3 项为铜耗, 其余 3 项为铁耗, 铜耗和铁耗主要由定子电流的基波分量 和总磁通确定。根据式(7), 电磁转矩 $T_{\mathrm{e}}$ 及电能损耗 $P_{\text {loss }}$ 为:

$$
\begin{gathered}
T_{\mathrm{e}}=\frac{3}{2} p\left[\psi_{\mathrm{m}} i_{q \mathrm{~m}}-\left(L_{d}-L_{q}\right) i_{d \mathrm{~m}} i_{q \mathrm{~m}}\right] \\
P_{\mathrm{loss}}=P_{\mathrm{Cu}}+P_{\mathrm{Fe}}
\end{gathered}
$$

式中, $P_{\mathrm{Cu}}$ 一铜耗, $P_{\mathrm{Fe}}$ 一铁耗。结合式(5)-(6)和 式(7), 稳态条件下铜耗和铁耗分别为: 


$$
\begin{gathered}
P_{\mathrm{Cu}}=\frac{3}{2} R_{\mathrm{s}}\left[\left(i_{d \mathrm{~m}}-\frac{\omega_{\mathrm{e}} L_{q} i_{q \mathrm{~m}}}{R_{c}}\right)^{2}+\left(i_{q \mathrm{~m}}-\frac{\omega_{\mathrm{e}}\left(\psi_{\mathrm{m}}-L_{d} i_{d \mathrm{~m}}\right.}{R_{c}}\right)^{2}\right] \\
P_{\mathrm{Fe}}=\frac{3}{2} R_{c}\left[\left(\frac{\omega_{\mathrm{e}} L_{q} i_{q \mathrm{~m}}}{R_{c}}\right)^{2}+\left(\frac{\omega_{\mathrm{e}}\left(\psi_{\mathrm{m}}-L_{d} i_{d \mathrm{~m}}\right.}{R_{c}}\right)^{2}\right]
\end{gathered}
$$

通常永磁同步风力发电机的电磁转矩表达式为:

$$
T_{\mathrm{g}}=\frac{3}{2} p\left[\psi_{\mathrm{m}} i_{q \mathrm{~s}}-\left(L_{d}-L_{q}\right) i_{q \mathrm{~s}} i_{d \mathrm{~s}}\right]
$$

对比式(8)和式(12), 式(12)忽略了铁耗电流。

\section{2. 最小功率损耗电流}

稳态条件下, 电磁转矩 $T_{\mathrm{e}}$ 和电角速度 $\omega_{\mathrm{e}}$ 可以看做是常 量。将式(8)代入式(9), 并结合式(10)和(11), 消去一个变 量, 可知总的电能损耗 $P_{\text {loss }}$ 是关于 $i_{d \mathrm{~m}}$ 和 $i_{q \mathrm{~m}}$ 的函数。若对 $P_{\text {loss }}$ 求导求解非常繁琐 $[16,17]$ 。本文采用拉格朗日乘子法和牛 顿迭代法求取给定参考电磁转矩 $T_{\mathrm{e} \text { ref }}$ 情况下, 最小电能损

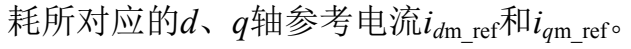

拉格朗日函数定义为 [18]:

$$
L\left(i_{d \mathrm{~m}}, i_{q \mathrm{~m}}\right)=P_{\text {loss }}+\lambda\left(T_{\mathrm{e}}\left(i_{d \mathrm{~m}}, i_{q \mathrm{~m}}\right)-T_{\text {e_ref }}\right)
$$

对拉格朗日函数 $L$ 求偏导, 并令各偏导数等于 0 , 可得:

$$
\begin{gathered}
\frac{\partial L}{\partial i_{d \mathrm{~m}}}=\frac{\partial P_{\text {loss }}}{\partial i_{d \mathrm{~m}}}+\lambda \frac{\partial T_{\mathrm{e}}}{\partial i_{d \mathrm{~m}}}=0 \\
\frac{\partial L}{\partial i_{q \mathrm{~m}}}=\frac{\partial P_{\text {loss }}}{\partial i_{q \mathrm{~m}}}+\lambda \frac{\partial T_{\mathrm{e}}}{\partial i_{q \mathrm{~m}}}=0 \\
\frac{\partial L}{\partial \lambda}=T_{\mathrm{e}}-T_{\mathrm{e}_{-} \text {ref }}=0
\end{gathered}
$$

联立式(14)和(15)消去 $\lambda$, 可得:

$$
f\left(i_{d \mathrm{~m}}, i_{q \mathrm{~m}}\right)=\frac{\partial P_{\text {loss }}}{\partial i_{d \mathrm{~m}}} \frac{\partial T_{\mathrm{e}}}{\partial i_{q \mathrm{~m}}}-\frac{\partial P_{\text {loss }}}{\partial i_{q \mathrm{~m}}} \frac{\partial T_{\mathrm{e}}}{\partial i_{d \mathrm{~m}}}=0
$$

式中,

$$
\begin{gathered}
\frac{\partial P_{\text {loss }}}{\partial i_{d \mathrm{~m}}}=k_{11} i_{d \mathrm{~m}}+k_{12} i_{q \mathrm{~m}}+k_{13} \\
\frac{\partial P_{\text {loss }}}{\partial i_{q \mathrm{~m}}}=k_{21} i_{d \mathrm{~m}}+k_{22} i_{q \mathrm{~m}}+k_{23} \\
\frac{\partial T_{\mathrm{e}}}{\partial i_{d \mathrm{~m}}}=k_{t d} i_{q \mathrm{~m}} \\
\frac{\partial T_{\mathrm{e}}}{\partial i_{q \mathrm{~m}}}=k_{t q}+k_{t d} i_{d \mathrm{~m}}
\end{gathered}
$$

式(18)-(21)中系数 $k_{i j}(i=1,2, j=1,2,3), k_{t d}, k_{t q}$ 的值 见附表1。将式(18)-(21)代入式(17)可得:

$$
\begin{aligned}
& f\left(i_{d \mathrm{~m}}, i_{q \mathrm{~m}}\right)=k_{11} k_{t d} i_{d \mathrm{~m}}^{2}-k_{22} k_{t d} i_{q \mathrm{~m}}^{2}+\left(k_{12}-k_{21}\right) k_{t d} i_{d \mathrm{~m}} i_{q \mathrm{~m}} \\
& +i_{d \mathrm{~m}}\left(k_{11} k_{t q}+k_{13} k_{t d}\right)+i_{q \mathrm{~m}}\left(k_{12} k_{t q}-k_{23} k_{t d}\right)+k_{13} k_{t q}
\end{aligned}
$$

注意到 $k_{12}=k_{21}$, 等式右边第三项系数为 0 。再将式(8) 代入式(22), 并联立式(16), 得出:

$$
\begin{aligned}
& f\left(i_{d \mathrm{~m}}\right)=k_{11} k_{t d} i_{d \mathrm{~m}}^{2}-k_{22} k_{t d}\left(\frac{T_{\mathrm{e}_{-} \text {ref }}}{k_{t q}+k_{t d} i_{d \mathrm{~m}}}\right)^{2}+k_{13} k_{t q} \\
& +\left(\frac{T_{\mathrm{e} \_ \text {ref }}}{k_{t q}+k_{t d} i_{d \mathrm{~m}}}\right)\left(k_{12} k_{t q}-k_{23} k_{t d}\right)+i_{d \mathrm{~m}}\left(k_{11} k_{t q}+k_{13} k_{t d}\right)
\end{aligned}
$$

对 $f\left(i_{d \mathrm{~m}}\right)=0$ 通分, 变换后可得 $f_{1}\left(i_{d \mathrm{~m}}\right)=0$, 即

$$
\begin{aligned}
& f_{1}\left(i_{d \mathrm{~m}}\right)=\left(k_{12} k_{t q}-k_{23} k_{t d}\right)\left(k_{t q}+k_{t d} i_{d \mathrm{~m}}\right) T_{\mathrm{e}_{\_} \text {ref }}-k_{22} k_{t d} T_{\mathrm{e}_{\mathrm{e} \_ \text {ref }}}^{2} \\
& +\left(k_{t q}+k_{t d} i_{d \mathrm{~m}}\right)^{2}\left[k_{11} k_{t d} i_{d \mathrm{~m}}^{2}+k_{13} k_{t q}+i_{d \mathrm{~m}}\left(k_{11} k_{t q}+k_{13} k_{t d}\right)\right]=0
\end{aligned}
$$

如前所述, 对式(24)进行数值求解是困难的。在这里 采用牛顿迭代法, 方法如式(25)所示:

$$
i_{d \mathrm{~m}_{-} n+1}=i_{d \mathrm{~m}_{-} n}-\frac{f_{1}\left(i_{d \mathrm{~m}_{-} n}\right)}{f_{1}^{\prime}\left(i_{d \mathrm{~m}_{-} n}\right)} \quad(n=0,1,2 \cdots)
$$

取 $d$ 轴电流初始值 $i_{d \mathrm{~m} \_0}=0, \mathrm{n}$ 为迭代次数。实验证明 经过2-3次迭代即可达到较高的精度。

\section{3. 电流环控制结构设计}

电磁转矩电流分量 $i_{d \mathrm{~m}}, i_{q \mathrm{~m}}$ 难以检测。实际上, 可以测 量得到的是发电机输出端定子电流分量 $i_{d s}, i_{q \mathrm{~s}}$ 。因此, 需

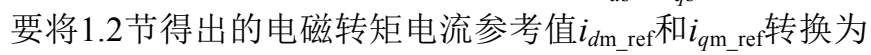

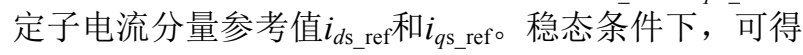

$$
i_{d \_\_ \text {ref }}=i_{d \mathrm{~m}_{-} \text {ref }}-\frac{\omega_{\mathrm{e}} L_{q} i_{q \mathrm{~m} \_ \text {ref }}}{R_{c}}
$$

$$
i_{q \mathrm{~s} \_ \text {ref }}=i_{q \mathrm{~m} \_ \text {ref }}-\frac{\left(\psi_{\mathrm{m}}-L_{d} i_{d \mathrm{~m}_{-} \text {ref }}\right) \omega_{\mathrm{e}}}{R_{c}}
$$

对式(1)和(2)进行拉普拉斯变换(各变量拉普拉斯函数 仍用其原有变量名替代), 并联立式(3)和(4), 可以得出:

$$
\begin{array}{r}
i_{q \mathrm{~m}}=-\frac{1}{R_{\mathrm{s}}+s L_{q}}\left[U_{q \mathrm{~s}}-\left(\psi_{\mathrm{m}}-L_{d} i_{d \mathrm{~s}}\right) \omega_{\mathrm{e}}+L_{d} i_{d \mathrm{c}} \omega_{\mathrm{e}}-R_{\mathrm{s}} i_{q \mathrm{c}}\right] \\
i_{d \mathrm{~m}}=-\frac{1}{R_{\mathrm{s}}+s L_{d}}\left[U_{d \mathrm{~s}}-L_{q} i_{q \mathrm{~s}} \omega_{\mathrm{e}}-\omega_{\mathrm{e}} L_{q} i_{q \mathrm{c}}-R_{\mathrm{s}} i_{d \mathrm{c}}\right]
\end{array}
$$

从上式可以看出, $d$ 轴和 $q$ 轴电流存在耦合, 因为等效 铁损电流不可测, 所以实现完全解耦非常困难。又因为 $i_{d c}$ 、 
$i_{q c}$ 值很小, 所以可将其看做扰动量, 由此可实现最大程度 解耦, 采用PI控制, 选取 $d 、 q$ 轴电压控制量为:

$$
\begin{gathered}
U_{q \mathrm{~s}}=G_{i q}\left(i_{q \mathrm{~s} \_ \text {ref }}-i_{q \mathrm{~s}}\right)+\left(\psi_{\mathrm{m}}-L_{d} i_{d \mathrm{~s}}\right) \omega_{\mathrm{e}} \\
U_{d \mathrm{~s}}=G_{i d}\left(i_{d \mathrm{~s}_{-} \text {ref }}-i_{\mathrm{ds}}\right)+L_{q} i_{q \mathrm{~s}} \omega_{\mathrm{e}}
\end{gathered}
$$

式中, $\mathrm{G}_{i d} 、 \mathrm{G}_{i q}$ 为PI控制器。进一步联立式(26)(27), 从式(28)(29)可以推导出:

$$
\begin{aligned}
i_{q \mathrm{~m}}= & \frac{-G_{i q} i_{q \mathrm{~m}_{-} \text {ref }}}{R_{\mathrm{s}}+s L_{q}-G_{i q}}+\frac{G_{i q}\left(\psi_{\mathrm{m}}-L_{d} i_{d \mathrm{~m}_{-} \text {ref }}\right) \omega_{\mathrm{e}}}{R_{c}\left(R_{\mathrm{s}}+s L_{q}-G_{i q}\right)} \\
& -\frac{i_{q c}\left(G_{i q}-R_{\mathrm{s}}\right)+L_{d} i_{d c} \omega_{\mathrm{e}}}{R_{\mathrm{s}}+s L_{q}-G_{i q}}
\end{aligned}
$$

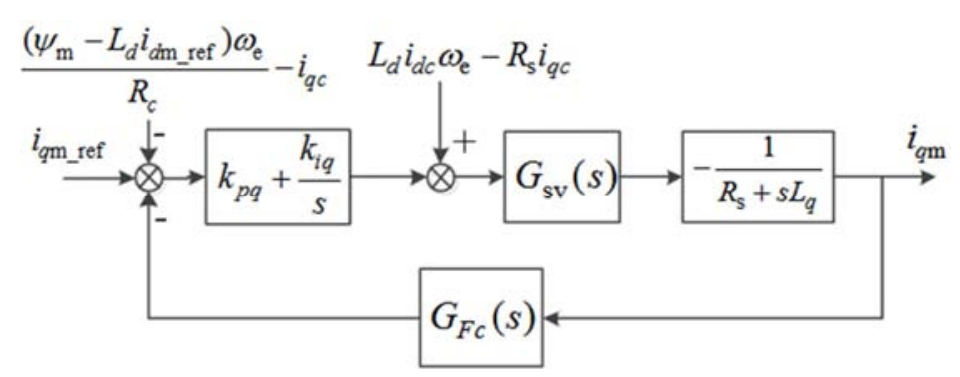

图2 q轴电流环控制框图。
同样以 $q$ 轴电流为研究对象, 忽略扰动项, 考虑主环 路, 则 $\mathrm{i}_{q \mathrm{~m} \_ \text {ref }}$ 到 $\mathrm{i}_{q \mathrm{~m}}$ 的开环传递函数为

$$
G_{c}=\left(k_{p q}+\frac{k_{i q}}{s}\right)\left(\frac{1}{s T_{s}+1} \cdot \frac{-T_{d} s / 2+1}{T_{d} s / 2+1}\right)\left(-\frac{1}{R_{\mathrm{s}}+s L_{q}}\right)\left(\frac{1}{s T_{F c}+1}\right)
$$

式中, 期望开环截止频率 $\omega_{c}^{*}$ 和相位裕度 $\varphi_{c m}^{*}$ 应该满 足[19]

$$
\begin{gathered}
\left|G_{c}\left(j \omega_{c}^{*}\right)\right|=1 \\
\varphi_{c m}^{*}=\angle G_{c}\left(j \omega_{c}^{*}\right)-(-\pi)
\end{gathered}
$$

联立式(36)和(37), 计算得到 $k_{p q}, k_{i q}$ 的解析值为:

$$
\begin{gathered}
k_{p q}=-A_{q_{-} \mathrm{amp}} \sqrt{\frac{1}{1+Q_{q c}}} \\
k_{i q}=-A_{q_{-} \mathrm{amp}} \omega_{c}^{*} \sqrt{\frac{Q_{q c}}{1+Q_{q c}}}
\end{gathered}
$$

\section{4. 电流环PI控制器参数选取}

$$
\begin{aligned}
i_{d \mathrm{~m}}= & \frac{-G_{i d} i_{d \mathrm{~m}_{-} \text {ref }}}{R_{\mathrm{s}}+s L_{d}-G_{i d}}+\frac{G_{i d} L_{q} i_{q \mathrm{~m}_{-} \text {ref }} \omega_{\mathrm{e}}}{R_{c}\left(R_{\mathrm{s}}+s L_{d}-G_{i d}\right)} \\
& -\frac{i_{d c}\left(G_{i d}-R_{\mathrm{s}}\right)-L_{q} i_{q c} \omega_{\mathrm{e}}}{R_{\mathrm{s}}+s L_{d}-G_{i d}}
\end{aligned}
$$

由此可得出, 当求得磁化电流参考值 $i_{d \mathrm{~m} \_r e f}$ 和 $i_{q \mathrm{~m} \_ \text {ref }}$ 后, 按式(30)和(31)所得电压值控制发电机输出端电压, 则 $i_{d \mathrm{~m}}$ 、 $i_{q \mathrm{~m}}$ 与其参考值之间满足式(32)和(33)描述的传递函数关系。 考虑到SVPWM电压源逆变器传递函数可以等效为:

$$
G_{\mathrm{sv}}=\frac{1}{s T_{s}+1} e^{-s T_{d}} \approx \frac{1}{s T_{s}+1} \cdot \frac{-\frac{T_{d}}{2} s+1}{\frac{T_{d}}{2} s+1}
$$

式中, $T_{s}$ —逆变器控制周期; $T_{d}$ 一延迟时间。以 永磁同步发电机 $q$ 轴电流为研究对象, $d$ 轴电流控制与 $q$ 轴 相同, 电流环控制结构图如图2所示。采样和滤波环节等 效为一阶低通滤波器 $G_{F c}$, 它的时间常数为 $T_{F c}$ 。

$$
\begin{gathered}
A_{q_{-} \mathrm{amp}}=\sqrt{\left[R_{\mathrm{s}}^{2}+\left(\omega_{c}^{*} L_{q}\right)^{2}\right]\left[1+\left(\omega_{c}^{*} T_{s}\right)^{2}\right]\left[1+\left(\omega_{c}^{*} T_{F c}\right)^{2}\right]} \\
Q_{q c}=\tan ^{2}\left(\varphi_{c m}^{*}-\pi-Q_{q_{-} \text {phase }}\right) \\
Q_{q_{-} \text {phase }}=\operatorname{arctg}\left(\frac{\omega_{c}^{*} L_{q}}{R}\right)+\operatorname{arctg}\left(\omega_{c}^{*} T_{s}\right) \\
+\operatorname{arctg}\left(\omega_{c}^{*} T_{F c}\right)+\operatorname{arctg}\left(\frac{T_{d} \omega_{c}^{*}}{1-\left(\frac{T_{d}}{2} \omega_{c}^{*}\right)^{2}}\right)
\end{gathered}
$$

校正后系统开环截止频率 $\omega_{c}^{*}$ 应该大于校正前电流环 截止频率 $\sqrt{\left(1-R_{\mathrm{s}}^{2}\right) / L_{q}} \mathrm{rad} / \mathrm{s}$ 。为减小信号失真, 系统闭环 带宽应该大于定子绕组电信号的最大频率 $\omega_{c \max }$, 而闭环 带宽通常为开环截止频率的1.4-1.6倍。另外, 逆变器开关 频率应处于系统高频段, 以避免开关谐波的干扰[20]。所 以, 开环截止频率取值范围为 $\left(\max \left(\sqrt{\left(1-R_{\mathrm{s}}^{2}\right) / L_{q}}, \omega_{c \text { max }}\right), \frac{2 \pi}{1.6} \times \frac{1}{10 T_{s}}\right) 。$

电机控制中, 常用PI控制器的零点对消定子电流传递 函数的极点, 将电流环整定为过阻尼系统, 以避免过大电 
流超调的冲击, 即 $\mathrm{PI}$ 控制其参数设计为 $k_{p q} R_{\mathrm{s}}=k_{i q} L_{q}$ 。结 合式(37), 此时电流环相位裕度为

$$
\varphi_{c m}=\frac{\pi}{2}-\operatorname{arctg}\left(\omega_{c}^{*} T_{s}\right)-\operatorname{arctg}\left(\omega_{c}^{*} T_{F c}\right)-\operatorname{arctg}\left(\frac{T_{d} \omega_{c}^{*}}{1-\left(\frac{T_{d}}{2} \omega_{c}^{*}\right)^{2}}\right)
$$

相位裕度主要影响系统的动态响应特性, 包括调节时 间、超调量和振荡等。工程上常将相位裕度设定为 $\pi / 4$ 左
右, 但不应超过式(43)的最大相位裕度, 否则稳态跟踪性 能明显变差, 甚至PI控制器无法满足设计要求。

根据附表 2 中提供的风力发电机数据配置 $q$ 轴电流环 PI 控制器, 取截止频率 $\omega_{c}^{*}=3000 \mathrm{rad} / \mathrm{s}$, 相位裕度 $\varphi_{c m}=56^{\circ}$, 校正前后系统开环传递函数Bode图如图3(a) 所示。由图可以看出, 校正前 $\mathrm{q}$ 轴电流环相位裕度为 $-112^{\circ}$, 系统不稳定。校正后, 其单位阶跃响应如图3(b)中实线所 示, 上升时间 $0.6 \mathrm{~ms}$, 峰值时间 $0.9 \mathrm{~ms}$, 超调量 $11.75 \%$ 。

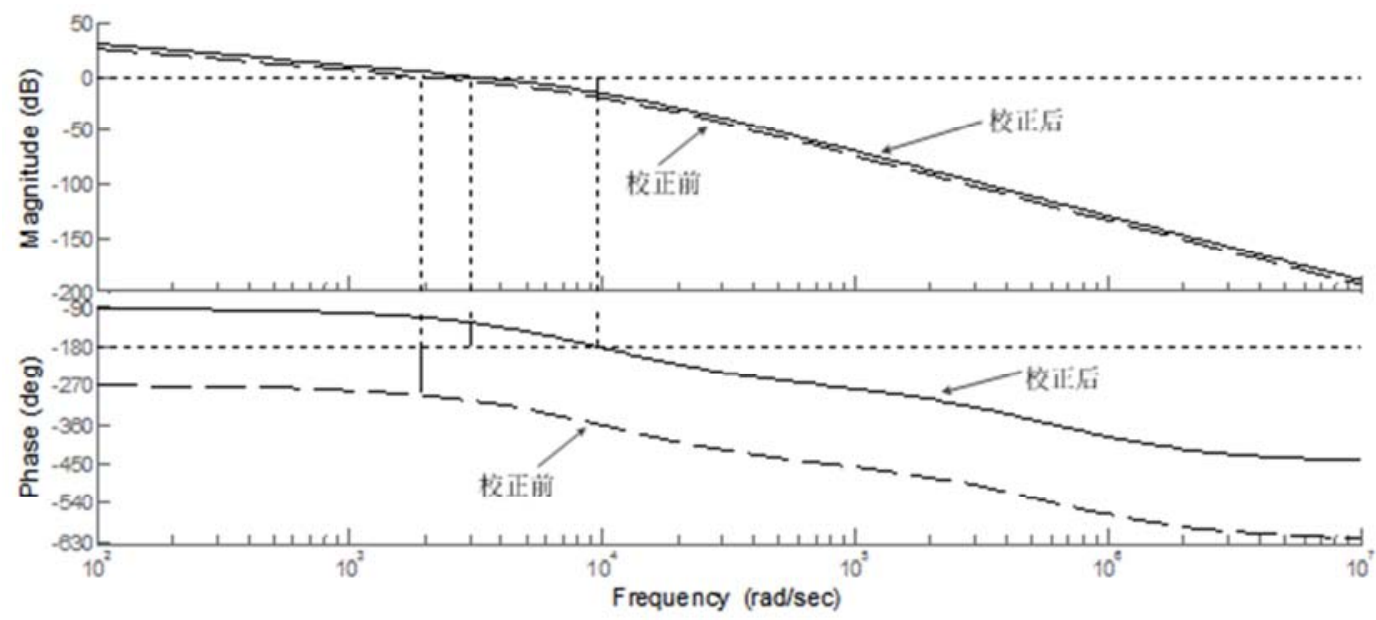

(a) $q$ 轴电流环开环传递函数校正前后伯德图

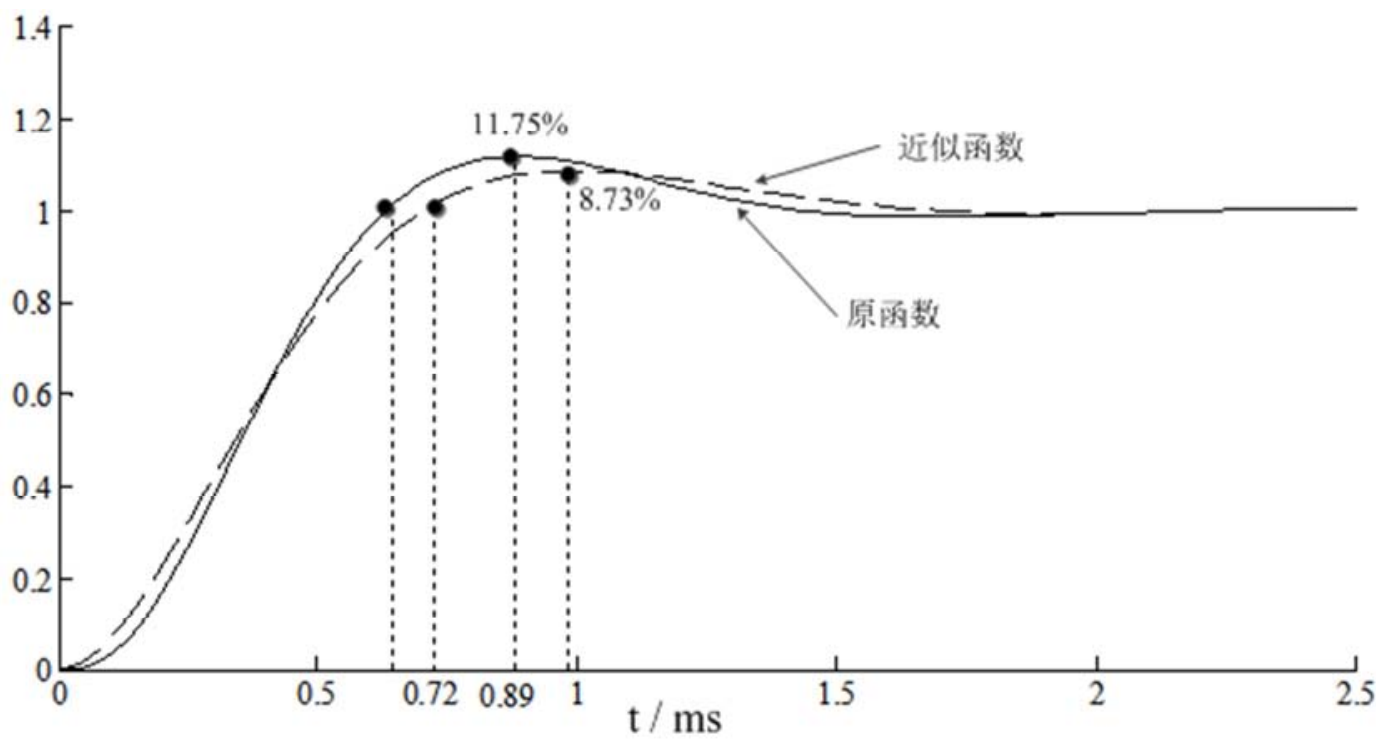

(b) $q$ 轴电流环闭环阶跃响应

图3 q轴电流环频率特性和响应曲线。

逆变器控制周期、延迟以及采样滤波环节的时间常数 都非常小, 因此, 将小惯性环节近似叠加为一个延时, 取 $T_{c}=T_{s}+T_{d} / 2+T_{F c}$ 。同时, 由于大型风机的惯量大, 电 磁转矩的调节远远快于风机转速的调节。因此, 可以对电 流环进行简化。 $d$ 轴和 $q$ 轴电流环传递函数结构相同, 按 1.4 节所述的方法配置 $d$ 轴电流环PI控制器参数, 使其具有与 $q$ 轴电流环相同的动态性能, 则 $d$ 轴和 $q$ 轴电流环闭环传递函 数近似为:

$$
G_{q}=G_{d}=\frac{1}{s^{2} \tau_{i}+\tau_{i} s / T_{c}+1}
$$

式中, $\tau_{i}=-R_{\mathrm{S}} T_{c} / k_{i q}$ 。 $q$ 轴电流环近似闭环传递函数 单位阶跃响应如图3(b)中虚线所示, 可以看出其与原函数 单位阶跃响应拟合度非常高, 上升与峰值时间非常接近。 


\section{3. 功率环分析}

\section{1. 风力发电机功率环模型}

风力发电机系统风力机转矩和运动方程分别为

$$
\begin{aligned}
& T_{\text {tur }}=\frac{1}{2} \rho \pi R_{\mathrm{r}}^{3} C_{\mathrm{T}}(\lambda) V^{2} \\
& J \frac{d \omega_{\mathrm{r}}}{d t}=T_{\text {tur }}-i T_{\mathrm{e}}-T_{\mathrm{f}}
\end{aligned}
$$

式中, $T_{\text {tur }}$ 风力机转矩; $R_{\mathrm{r}}$ 一轮半径; $C_{\mathrm{T}}$ 转矩系数 (其是关于叶尖速比 $\lambda$ 的函数, $\lambda=\frac{R_{\mathrm{r}} \omega_{\mathrm{r}}}{V}$ ); $\omega_{\mathrm{r}}$ 风轮转速, $V$ 一一速; $J$ 为转动惯量; $T_{\mathrm{f}}$ 一摩擦力力矩, $T_{\mathrm{f}}=B \omega_{\mathrm{r}} ; \mathrm{B}$ 一一等效摩擦系数; $i$ 一一齿轮比。

对风力机转矩方程进行小信号分析[21], 式(45)线性 化可得:

$$
\Delta T_{\text {tur }}=\frac{\rho \pi R_{\mathrm{r}}^{3}}{2}\left(2 V C_{\mathrm{T}}-R_{\mathrm{r}} \omega_{\mathrm{r}} k_{\mathrm{T} \lambda}\right) \Delta V+\frac{\rho \pi R_{\mathrm{r}}^{4}}{2} V \cdot k_{\mathrm{T} \lambda} \Delta \omega_{\mathrm{r}}(47)
$$

式中, $k_{\mathrm{T} \lambda}=\frac{\partial C_{\mathrm{T}}}{\partial \lambda}$ 。

将式(46)线性化, 并将式(47)代入, 可得:

$$
\begin{gathered}
\Delta \omega_{\mathrm{r}}=\frac{\tau_{w} \rho \pi R_{\mathrm{r}}^{3}\left(2 V C_{\mathrm{T}}-R_{\mathrm{r}} \omega_{\mathrm{r}} k_{\mathrm{T} \lambda}\right)}{2 J\left(\tau_{w} s+1\right)} \Delta V-\frac{i \tau_{w}}{J\left(\tau_{w} s+1\right)} \Delta T_{\mathrm{e}} \\
\tau_{w}=\frac{2 J}{-\rho \pi R_{\mathrm{r}}^{4} V \cdot k_{\mathrm{T} \lambda}+2 \mathrm{~B}}
\end{gathered}
$$

永磁同步风力发电机电磁功率为:

$$
P_{\mathrm{e}}=T_{\mathrm{e}} \omega_{\mathrm{m}}
$$

式中, $\omega_{\mathrm{m}}$ 一发电机转速, $\omega_{\mathrm{m}}=i \omega_{\mathrm{r}}$ 。对式(50)线 性化, 并将式(48)代入，可得:

$$
\Delta P_{\mathrm{e}}=i \omega_{\mathrm{r}} \frac{\tau_{w}\left(\tau_{z} s+1\right)}{\tau_{z}\left(\tau_{w} s+1\right)} \Delta T_{\mathrm{e}}+\frac{i T_{\mathrm{e}} \tau_{w} \rho \pi R_{\mathrm{r}}^{3}\left(2 V C_{\mathrm{T}}-R_{\mathrm{r}} \omega_{\mathrm{r}} k_{\mathrm{T} \lambda}\right)}{2 J\left(\tau_{w} s+1\right)} \Delta V
$$

其中, $\tau_{z}=\frac{J \omega_{\mathrm{r}} \tau_{w}}{J \omega_{\mathrm{r}}-i T_{\mathrm{e}} \tau_{w}}$ 。

结合式(44)和式(51), 功率控制回路结构如图4所示, 其中 $G_{P c}$ 为一阶滤波器, $G_{p i}$ 为 $\mathrm{PI}$ 控制器。

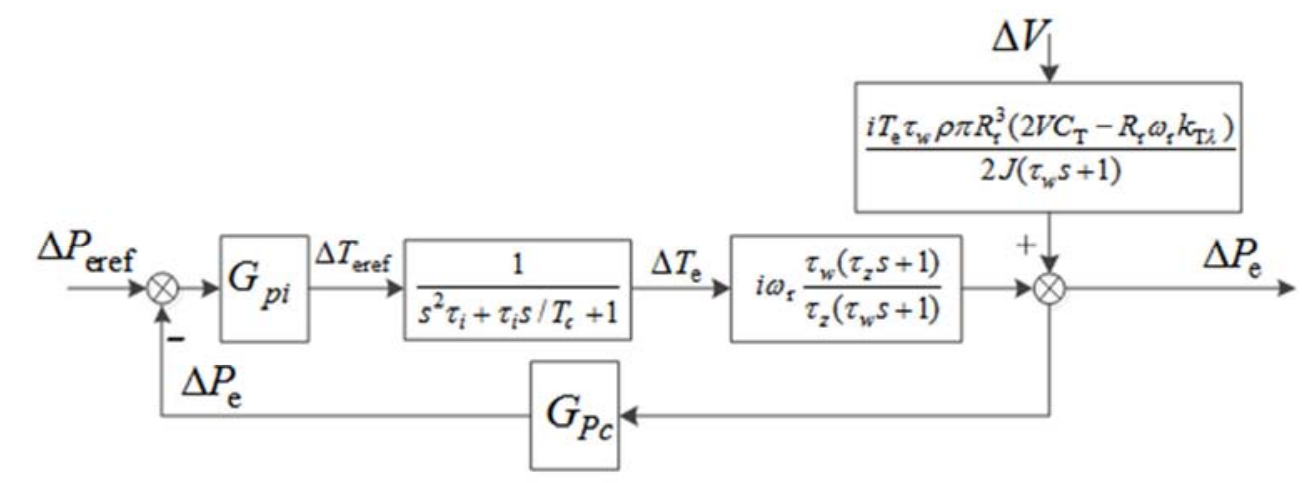

图4 线性化功率控制回路框图。

在实际系统中, 测量的是发电机输出功率 $P_{\mathrm{g}}$ 。在图 4 中, 若用 $\Delta P_{\mathrm{g}}$ 代替 $\Delta P_{\mathrm{e}}$, 相当于忽略了机组损耗, 实际上 是在追踪一个物理上不可能达到的最大功率点。所以, $\Delta P_{\mathrm{e}}$ 应该为

$$
\Delta P_{\mathrm{e}}=\Delta P_{\mathrm{g}}+\Delta P_{\mathrm{f}}+\Delta P_{\text {loss }}
$$

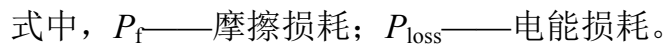

图4的线性化模型显示了工作点所决定的系统模型的 零、极点对机组性能的影响。因为 $\tau_{i} \ll \tau_{i} / T_{c}, \tau_{i} / T_{c}$ 远小 于功率环时间常数 $\tau_{w}$, 可以认为电流环对参考转矩的实 现是瞬间完成的。尽管可以通过设计功率环PI控制器使得 输出功率快速跟踪参考功率, 但由于风电机组较大的 $\tau_{w}$ 仍将使风轮转速 $\omega_{\mathrm{r}}$ 的调节有一定的延迟。 $\tau_{w}$ 与 $\tau_{z}$ 取决于工作点, 风力发电机组运行于功率系 数曲线的下降部分, 即 $k_{T \lambda}<0$, 以保证系统闭环传递函数 中不因 $\tau_{w}$ 而出现不稳定的正的极点。结合式(48)、(49)可 以看出, 电磁转矩的变化将会使发电机功率有一个跃升, 即 $i \omega_{\mathrm{r}} \Delta T_{\mathrm{e}}$ 。之后以指数律下降至新的稳定状态 $\Delta P_{\mathrm{e}}=i \omega_{\mathrm{r}} \tau_{w} \Delta T_{\mathrm{e}} / \tau_{z}$ 。

\section{2. 功率环控制器设计}

由图4得功率环开环传递函数为:

$G_{\mathrm{P}}=k_{p p}\left(1+\frac{1}{T_{i} s}\right) \cdot \frac{1}{T_{p} s+1} \cdot \frac{1}{s^{2} \tau_{i}+\tau_{i} s / T_{c}+1} \cdot \frac{i \omega_{\mathrm{r}} \tau_{w}\left(\tau_{z} s+1\right)}{\tau_{z}\left(\tau_{w} s+1\right)}$ 
令 $M_{G}$ 为未校正系统幅值, $\theta_{G}$ 为未校正系统相角, $\varphi_{p m}^{*}$ 为预期的相角裕度。加入PI控制器后, 在期望的截止 频率 $\omega_{c p}^{*}$ 处, 应该满足

$$
M_{G} e^{j \theta_{G}} \cdot \frac{k_{p p}\left(T_{i} \omega_{c p}^{*} j+1\right)}{T_{i} \omega_{c p}^{*} j}=1 \cdot e^{j\left(-\pi+\varphi_{p m}^{*}\right)}
$$

式中，

$$
\begin{gathered}
M_{G}^{2}=\frac{1}{1+\left(\omega_{c p}^{*} T_{p}\right)^{2}} \cdot \frac{1}{\left(1-\omega_{c p}^{*} \tau_{i}\right)^{2}+\left(\frac{\tau_{i}}{T_{c}} \omega_{c p}^{*}\right)^{2}} \\
. \frac{\left(i \omega_{\mathrm{r}} \tau_{w}\right)^{2}\left[\left(1+\omega_{c p}^{* 2} \tau_{z} \tau_{w}\right)^{2}+\left(\tau_{z}-\tau_{w}\right)^{2} \omega_{c p}^{*}\right]}{\tau_{z}^{2}\left(1+\omega_{c p}^{*} \tau_{w}^{2}\right)^{2}} \\
\theta_{G}=\operatorname{arctg}\left(-T_{p} \omega_{c p}^{*}\right)+\operatorname{arctg}\left(\frac{\tau_{i} \omega_{c p}^{*}}{T_{c} \tau_{i} \omega_{c p}^{*}-T_{c}}\right) \\
+\operatorname{arctg}\left(\frac{\left(\tau_{z}-\tau_{w}\right) \omega_{c p}^{*}}{1+\tau_{z} \tau_{w} \omega_{c p}^{*}}\right)
\end{gathered}
$$

式(54)中, 等式两边虚部与实部应该相等。所以, 可 以得出 $k_{p p}, T_{i}$ 的解分别为:

$$
\begin{gathered}
k_{p p}=-\frac{\cos \left(\theta_{G}-\varphi_{p m}^{*}\right)}{M_{G}} \\
T_{i}=\frac{\cos \left(\theta_{G}-\varphi_{p m}^{*}\right)}{\omega_{c p}^{*} \sin \left(\theta_{G}-\varphi_{p m}^{*}\right)}
\end{gathered}
$$

若选取 $T_{i}=\tau_{w}, T_{p}=\tau_{z}$, 则可得到

$$
\varphi_{p m}=\pi / 2-\operatorname{arctg}\left(\frac{\tau_{i} \omega_{c p}^{*}}{T_{c}-T_{c} \tau_{i} \omega_{c p}^{*}}\right)=\varphi_{p m_{-} \max }
$$

定义 $\varphi_{p m_{-} \text {max }}$ 为功率环相位裕度的最大值, 继续增加 相位裕度, PI控制器积分作用已不明显, 系统响应时间延 长, 稳定性能变差。所以, 相位裕度选取区间为 $\pi / 4<\varphi_{p m}<\varphi_{p m_{-} \max }$ 。同时, 为避免功率环串扰电流环, 截止频率 $\omega_{c p}$ 应该远小于电流环截止频率 $\omega_{c}$ 。

\section{4. 仿真实验}

选取西北某风场 $2 \mathrm{MW}$ 永磁同步风力发电机为研究对 象, 部分机组参数见附录。为进一步研究发电机损耗对机 组功率跟踪的影响, 将 $i_{d}=0$ 控制和MTPA控制方法与本文 提出的方法进行比较。另结合风力机功率系数和实验阶跃 响应数据, 选取 $\tau_{w}=27.32 s, \tau_{z}=11 \tau_{w}$, $k_{\mathrm{T} \lambda} \in\{-0.0045,-0.006\}$ (当 $\lambda \in\{8,9.75\}$ 时)。设定电流环时
间常数 $\tau_{i} / T_{c}=3.05 \times 10^{-4} \mathrm{~S}$, 功率环截止频率 $\omega_{c p}^{*}=500 \mathrm{rad} / \mathrm{s}$, 相位裕度 $\varphi_{p m}^{*}=60^{\circ}$ 。

\section{1. 损耗及功率点偏移分析}

以一恒定风速下风力发电机转速和电磁转矩作为参 考条件, $P_{\mathrm{Cu}} 、 P_{\mathrm{Fe}}$ 与电流 $i_{d \mathrm{~m}} 、 i_{q \mathrm{~m}}$ 满足式(10)和式(11)的关系, 铜耗、铁耗及总电损耗曲线如图 5 所示。与 $i_{d}=0$ 及MTPA两 种方式的发电机功率损耗比较, 这两种控制均忽略了铁耗 的存在。因此, 从图中可以看出, 其电磁转矩偏离了参考 转矩, 并且 $i_{d}=0$ 控制损耗功率最大, 其次是MTPA控制, $\mathrm{LMC}$ 控制损耗明显低于前两种控制, 比 $i_{d}=0$ 控制低 $42 \%$ 。

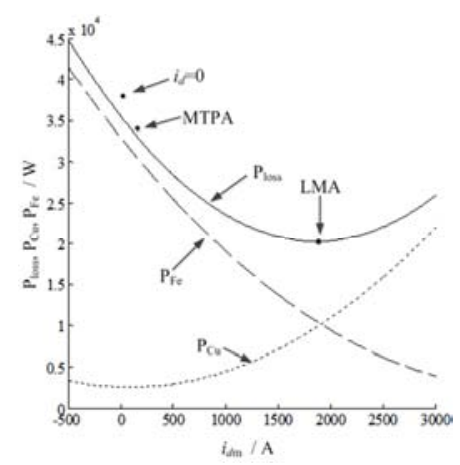

图5 $\mathrm{i}_{d}=0$, MTPA和LMC控制比较。

发电机损耗造成了发电机输出功率系数相对风机功 率系数的偏移, 其可表示为

$$
C_{g p}\left(\omega_{\mathrm{r}}, V\right)=\frac{P_{\mathrm{g}}}{P_{\mathrm{w}}}=C_{p}-\frac{\frac{3}{2} R_{\mathrm{s}}\left(i_{d \mathrm{~s}}^{2}+i_{q \mathrm{~s}}^{2}\right)+\frac{3}{2} R_{\mathrm{c}}\left(i_{d \mathrm{c}}^{2}+i_{q \mathrm{c}}^{2}\right)+B \omega_{\mathrm{r}}^{2}}{P_{\mathrm{w}}}
$$

图6为LMC、 $i_{d}=0 、 \mathrm{MTPA}$ 三种控制方式的发电机输出 功率系数与叶尖速比的关系曲线。由于大型风力发电机损 耗功率相对于风力机输出功率很小。因此, 最大功率系数 点在以叶尖速比为横坐标的方向上偏移较小, 可以认为风 力机与发电机共同达到最大功率输出点。三种方式均显示 损耗造成了机组输出功率的下降, 其中, LMC方法功率下 降程度最低, $i_{d}=0$ 控制与MTPA控制功率下降均明显大于 LMC方法。

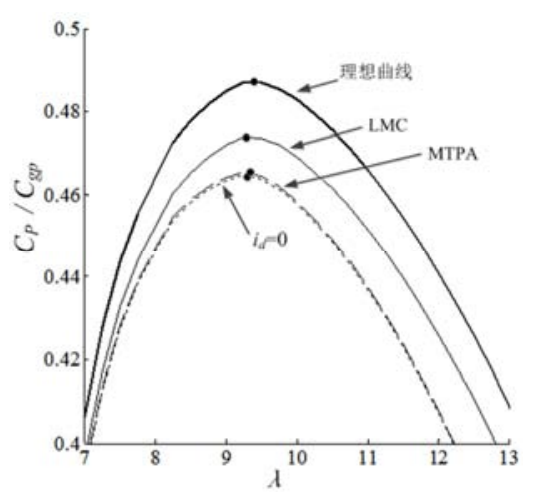

图6 风机与发电机功率系数曲线。 


\section{2. 连续风况运行分析}

根据风场提供的数据, 建立 $4-9 \mathrm{~m} / \mathrm{s}$ 风速模型, 湍流强 度 0.12 , 时长 $3000 \mathrm{~s}$, 如图7所示。在最优功率控制时, 控 制参数相同的情况下, 对LMC、 $i_{d}=0$ 和MTPA控制方式下, 风力发电机组运行情况进行对比和分析。发电机输出功率、 输出端交直轴电流分别如图8(a)-(c)所示。从图中可以看出,
三种方式均能较好地跟踪最大风能。其中, LMC控制方式 下的输出功率略高于其他两种控制方式, 在整个风速区间 内, 平均输出功率 $0.7929 \mathrm{MW}$ 。 $i_{d}=0$ 控制与MTPA控制方 式平均输出功率分别为 $0.7688 \mathrm{MW}$ 和 $0.77 \mathrm{MW}$ 。采用最小 损耗电流控制, 输出功率相对提升 $2 \%$ 左右。

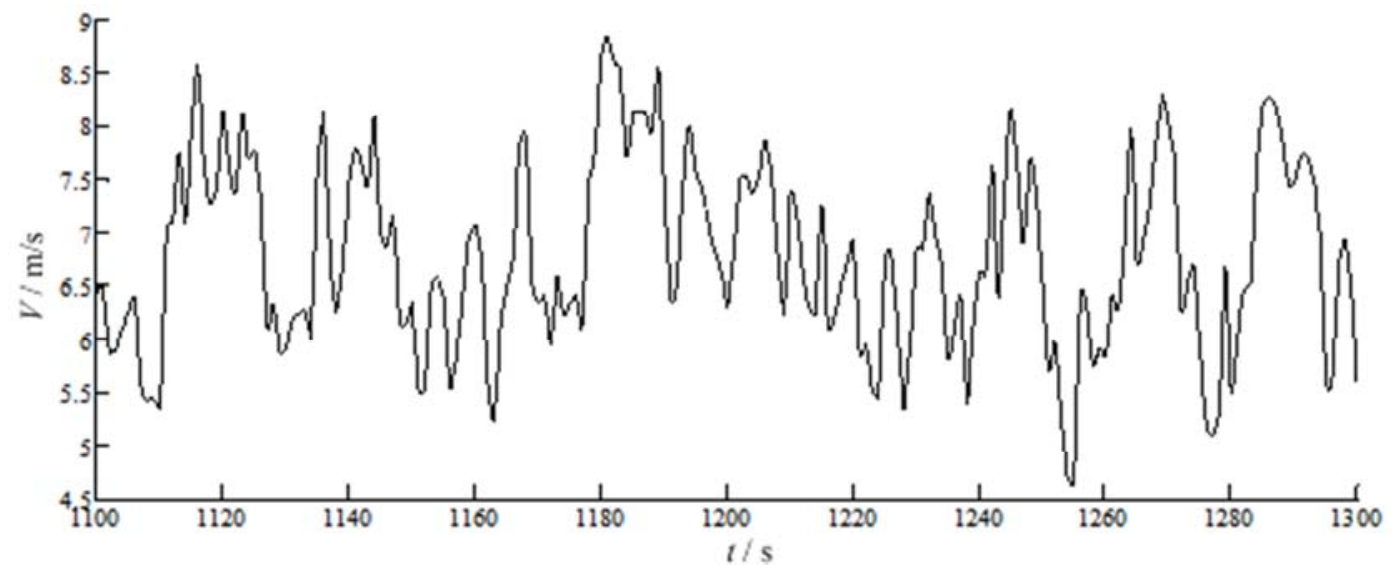

图7 风速序列。

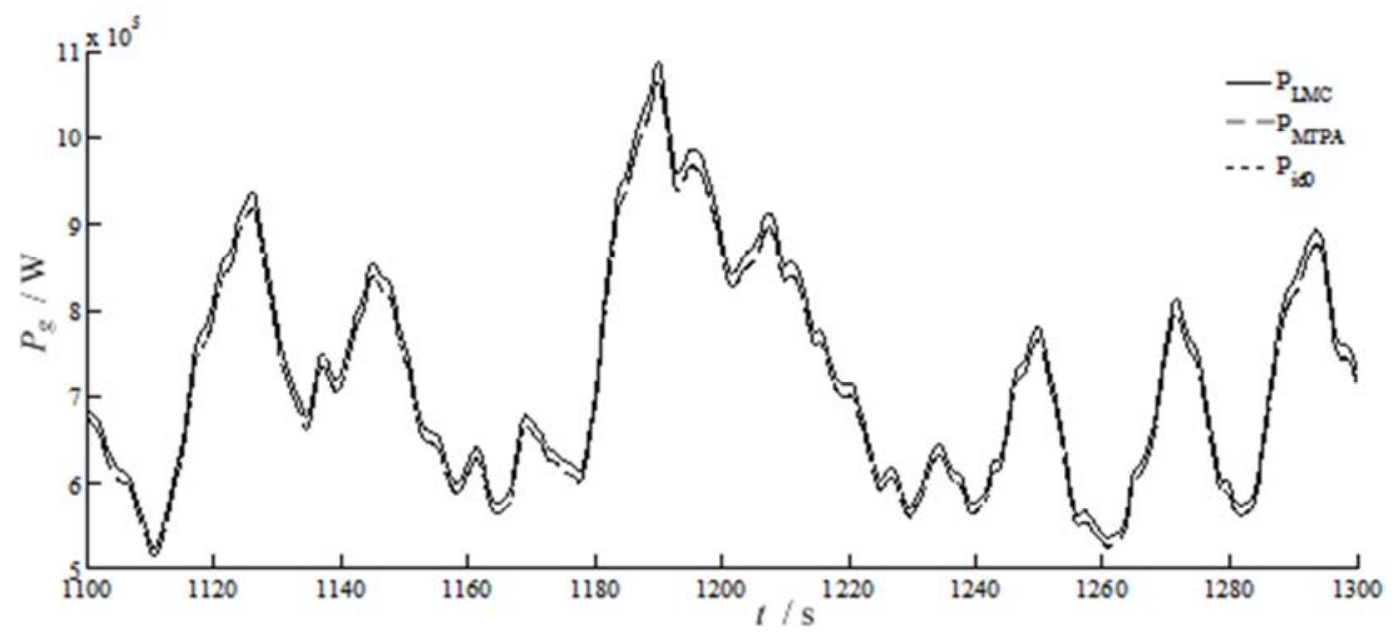

(a)发电机输出功率

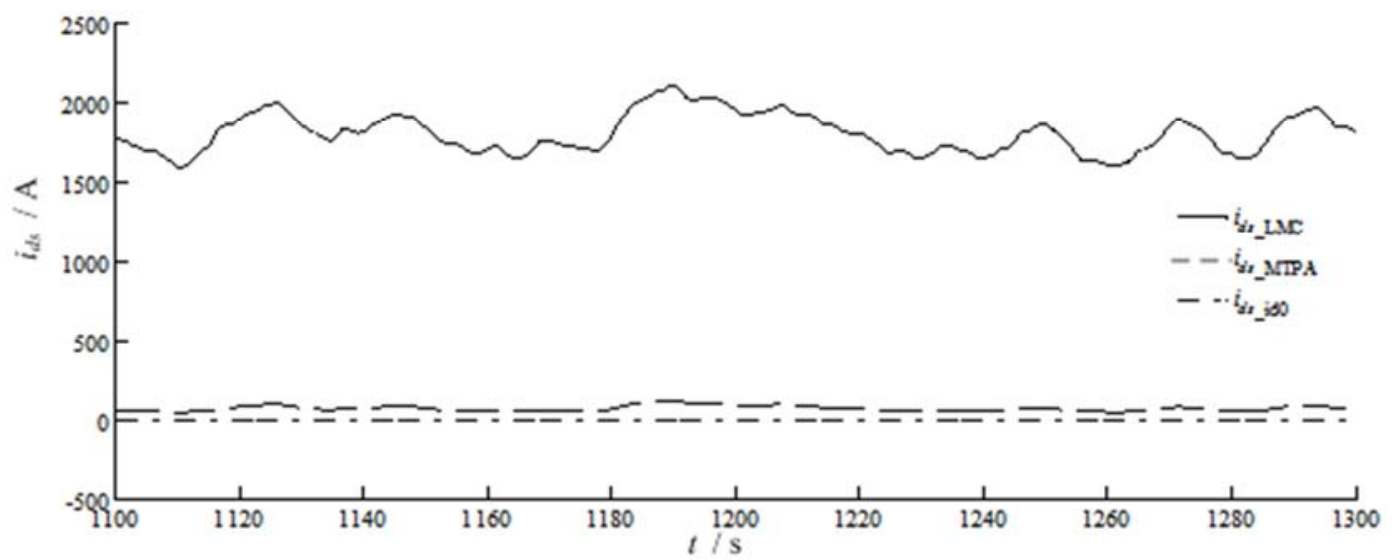

(b)发电机输出端直轴电流 


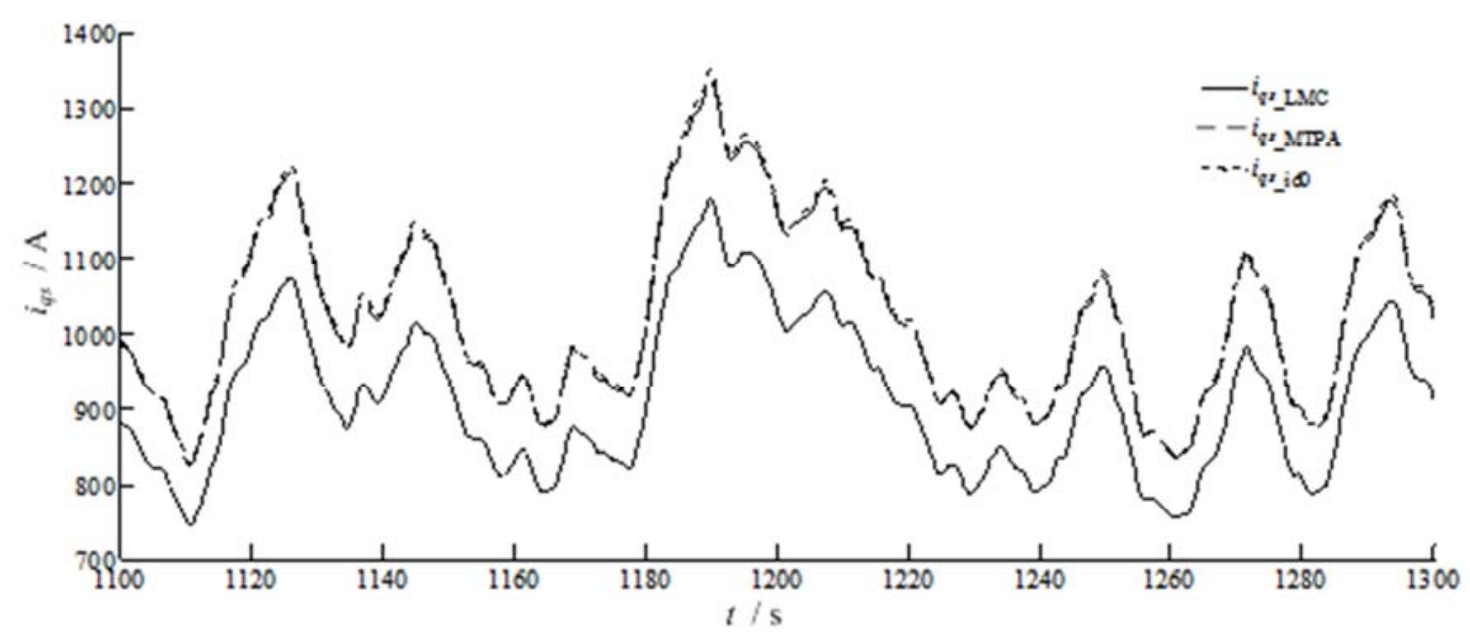

(c)发电机输出端交轴电流

图8 发电机输出功率、电流分布图。

因为等效铁耗电流很小, 所以交、直轴电磁感应电流 $i_{d \mathrm{~m}} 、 i_{q \mathrm{~m}}$ 与发电机输出端电流趋势基本相同。对于三种控制 方式, 交轴电流相差明显小于直轴电流之间的差值。这也 符合式(8)中电流与电磁转矩的规律。因为发电机定子绕组 电阻很小, 同时, $i_{d}=0$ 控制和MTPA控制方式未考虑铁耗, 所以他们的直轴电流远小于LMC控制方式。

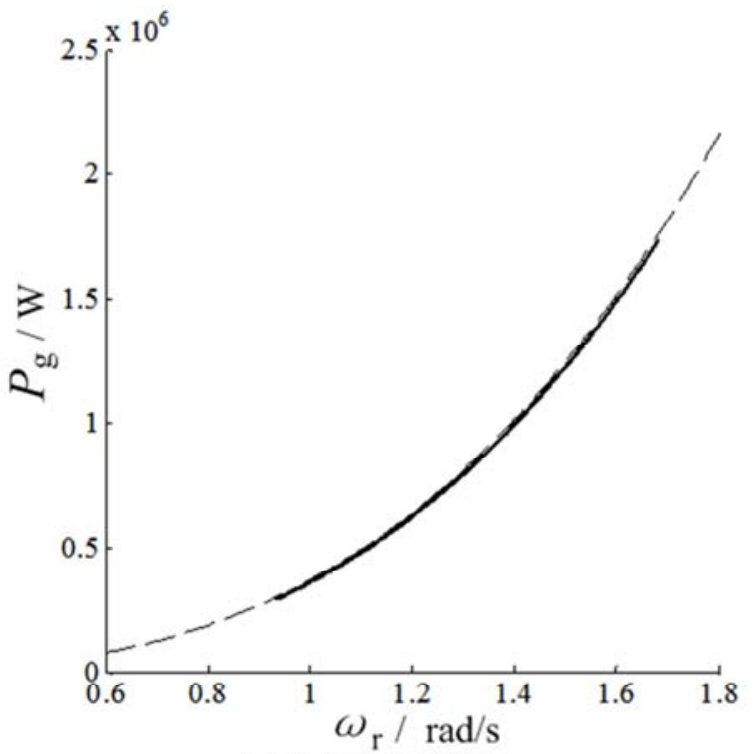

(a)输出功率轨迹图
图9(a)显示LMC控制方式输出功率紧密地跟踪了最 优功率轨迹, 因为损耗的存在, 所以略有向下偏移。图9(b) 显示运行区间内, $\mathrm{LMC}$ 控制方式风力机工作点的功率系数 集中于最大功率系数附近。这也证明了文中采用频域分析 法设计PI控制器可以使风力发电机拥有较好的动态性能。

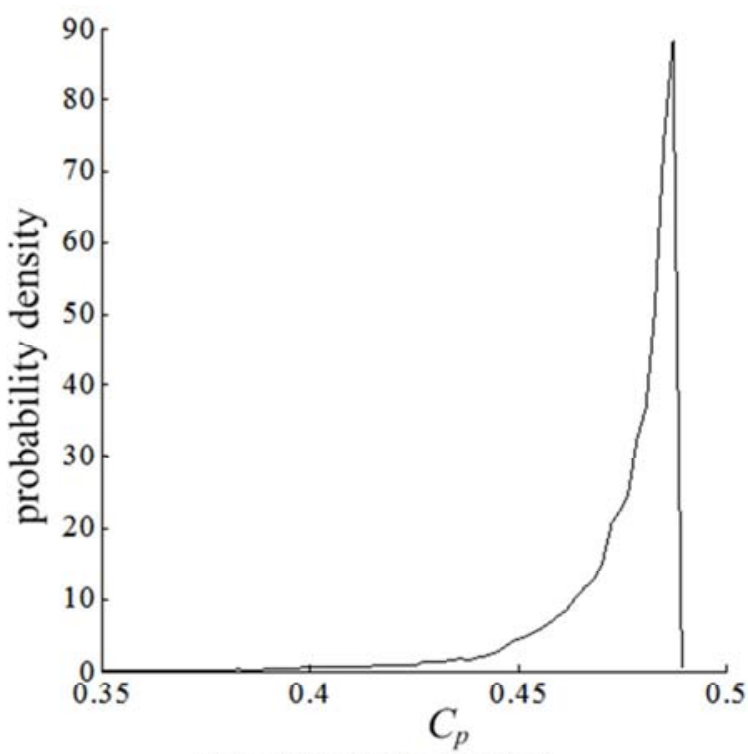

(b)风能利用系数概率密度

图9 风湍流响应功率跟踪。

\section{5. 结论}

永磁同步风力发电机功率损耗虽然所占比重很小, 但 是累计年发电量损失却非常可观。本文以 $2 \mathrm{MW}$ 永磁同步 风力发电机为对象, 利用小信号法建立了风力发电机组传 递函数模型, 利用频域分析法设计了电流环和功率环PI控 制器, 将所提出的最小损耗控制方式与设计的PI控制器应 用于非线性风力发电机模型，并与常用的 $i_{d}=0$ 控制和 MTPA控制进行了比较, 验证了减小发电机损耗和PI控制 器设计的有效性。
实验结果显示在湍流风速下, 永磁同步风力发电机具 有良好的动态性能, 能够跟踪最大功率点。控制器设计和 损耗影响分析对大型风力发电机控制提供了一定参考。今 后的研究将扩展非线性控制方式补偿机组损耗, 加快响应 速度的同时减小机组的载荷。

\section{基金项目}

陕西省重点研发计划项目(2017GY-061)。 
附录. 仿真系统参数

表1 拉格朗日函数L中的参数。

\begin{tabular}{ll}
\hline$k_{11}=3 R_{\mathrm{s}}+\frac{3 L_{d}^{2} \omega_{\mathrm{e}}^{2}}{R_{\mathrm{c}}}+\frac{3 R_{\mathrm{s}} L_{d}^{2} \omega_{\mathrm{e}}^{2}}{R_{\mathrm{c}}^{2}}$ & $k_{12}=\frac{3 R_{\mathrm{s}} L_{d} \omega_{\mathrm{e}}}{R_{\mathrm{c}}}-\frac{3 R_{\mathrm{s}} L_{q} \omega_{\mathrm{e}}}{R_{\mathrm{c}}}$ \\
$k_{13}=-\frac{3 L_{d} \omega_{\mathrm{e}}^{2} \psi_{\mathrm{m}}}{R_{\mathrm{c}}}-\frac{3 R_{\mathrm{s}} L_{d} \omega_{\mathrm{e}}^{2} \psi_{\mathrm{m}}}{R_{\mathrm{c}}^{2}}$ & $k_{21}=\frac{3 R_{\mathrm{s}} L_{d} \omega_{\mathrm{e}}}{R_{\mathrm{c}}}-\frac{3 R_{\mathrm{s}} L_{q} \omega_{\mathrm{e}}}{R_{\mathrm{c}}}$ \\
$k_{22}=3 R_{\mathrm{S}}+\frac{3 L_{q}^{2} \omega_{\mathrm{e}}^{2}}{R_{\mathrm{c}}}+\frac{3 R_{\mathrm{s}} L_{q}^{2} \omega_{\mathrm{e}}^{2}}{R_{\mathrm{c}}^{2}}$ & $k_{23}=-\frac{3 R_{\mathrm{s}} \omega_{\mathrm{e}} \psi_{\mathrm{m}}}{R_{\mathrm{c}}}$ \\
$k_{t d}=\frac{3}{2} p\left(L_{q}-L_{d}\right)$ & $k_{t q}=\frac{3}{2} p \psi_{\mathrm{m}}$ \\
\hline
\end{tabular}

表2 2MW永磁同步风力发电机主要参数。

\begin{tabular}{|c|c|c|c|}
\hline 参数 & 数值 & 参数 & 数值 \\
\hline 风轮半径(m) & 50 & 定子绕组电阻 $(m \Omega)$ & 1.5 \\
\hline 风轮转动惯量 $\left(\mathrm{kg} \cdot \mathrm{m}^{2}\right)$ & 1144940 & 定子交轴电感(mH) & 0.5 \\
\hline 齿轮比 & 83 & 定子直轴电感 $(\mathrm{mH})$ & 0.39 \\
\hline 传动轴等效摩擦系数 (N.m.s) & 600 & 永磁体磁链 $(\mathrm{Wb})$ & 1.49 \\
\hline 发电机功率 $P_{\mathrm{g}}(\mathrm{MW})$ & 2 & 极对数 & 3 \\
\hline 发电机转动惯量 $\left(\mathrm{kg} \cdot \mathrm{m}^{2}\right)$ & 77.3 & 铁耗等效电阻 $(\Omega)$ & 15.7 \\
\hline 最佳叶尖速比 & 9.25 & 最大功率系数 & 0.4872 \\
\hline
\end{tabular}

\section{参考文献}

[1] Hossain M M, Ali M H. Future research directions for the wind turbine generator system[J]. Renewable and Sustainable Energy Reviews, 2015, 49: 481-489.

[2] Cheng Ming, Zhu Ying. The state of the art of wind energy conversion systems and technologies : A review $[\mathrm{J}]$. Energy Conversion and Management, 2014, 88: $332-347$.

[3] 李咸善, 徐浩, 杜于龙. 采用叶尖速比法和爬山搜索法 相结合的风力发电系统最大功率点跟踪研究 $[\mathrm{J}]$. 电力 系统保护与控制，2015，43(13)：66-72.

[4] Li Xianshan, Xu Hao, Du Yulong. Maximum power tracking of wind power generation system using the combination of tip speed ratio method and climbing search method[J]. Power System Protection and Control, 2015, 43(13): 66-72(in Chinese).

[5] Ghaffari A, Krstic M, Seshagiri S. Power optimization and control in wind energy conversion systems using extremum seeking $[\mathrm{J}]$. IEEE Transactions on Control Systems Technology, 2014, 22(5): 1684-1695.

[6] Nasiri M, Milimonfared J, Fathi S H. Modeling, analysis and comparison of TSR and OTC methods for MPPT and power smoothing in permanent magnet synchronous generator-based wind turbines[J]. Energy Conversion and Management, 2014, 86(5): 892-900.

[7] 李滨, 李岗. 考虑损耗功率的双馈风力发电系统最大风能 跟踪控制 $[J]$. 电机与控制应用，2015，42(8)：55-59.

[8] Li Bin, Li Lan. Maximum power point tracking control for doubly-fed wind energy conversion systems considering loss power[J]. Electric Machines Control Application, 2015, 42 (8): 55-59(in Chinese).

[9] De Kooning J D M, Vandoorn T L, Jan V D V, et al. Displacement of the maximum power point caused by losses in wind turbine systems[J]. Renewable Energy, 2016, 85(1): 273-280.

[10] Bonfiglio A, Delfino F, Invernizzi M, et al. Modeling and Maximum Power Point Tracking Control of Wind Generating Units Equipped with Permanent Magnet Synchronous Generators in Presence of Losses[J]. Energies, 2017, 10(102): $1-24$.

[11] Gorman S . Design of an efficient small wind-energy conversion system with an adaptive sensorless MPPT strategy[J]. Renewable Energy, 2016, 86(11): 280-291.

[12] Sun T, Wang J, Chen X. Maximum torque per ampere (MTPA) control for interior permanent magnet synchronous machine drives based on virtual signal injection[J]. IEEE Transactions on Power Electronics, 2015, 30(9): 5036-5045.

[13] Biel Z, Vittek J, Hrkel M. Permanent magnet synchronous motor loss minimization control strategies[A] . IEEE Elektro[C], Rajeck Teplice, Slovakia, 2012. 
[14] Cavallaro C, Ditommaso A O, Miceli R, et al. Efficiency enhancement of permanent-magnet synchronous motor drives by online loss minimization approaches[J]. IEEE Transactions on Industrial Electronics， 2005， 52(4): 1153-1160.

[15] Zhu Y, Cheng M, Hua W, et al. A novel maximum power point tracking control for permanent magnet direct drive wind energy conversion systems $[\mathrm{J}]$. Energies, 2012, 5(5) : 1398-1412.

[16] 赵勇, 冯延轫, 邱颖宁. 一种考虑损耗的风电系统MPPT 控制方法研究[J]. 太阳能学报, 2018, 39(11): 3239-3244.

[17] Zhao Yong, Feng Yanhui, Qiu Yingning. Research on a mppt control method for wind power system considering loss[J] Acta Energiae Solaris Sinica, 2018, 39(11): 3239-3244(in Chinese).

[18] 朱瑛, 程明, 花为, 等. 考虑损耗转矩的风力发电系统最 大风能跟踪控制 $[J]$. 中国电机工程学报, 2013, 33(19): $39-46$.

[19] Zhu Ying, Cheng Ming, Hua wei, et al. Maximum power point tracking control for wind energy conversion systems considering loss torque[J]. Proceedings of the CSEE, 2013, 33(19): 39-46(in Chinese).

[20] 戴中林. 一元四次整系数多项式的因式分解法 $[\mathrm{J}]$. 大学数 学, 2016, 32(6): 101-105.

[21] Dai Zhonglin. A quartic polynomial decomposition method in the real domain factod[J]. College Mathematics, 2016, 32(6): 101-105(in Chinese).
[22] 周志宏. 一元四次方程 2 种根式求解算法的精度分析 [J]. 长 江大学学报自然科学版：理工卷, $2009,6(3)$ : 5-6.

[23] Zhou Zhihong. Accuracy analysis of two root solution algorithms for quartic equation of one variable $[\mathrm{J}]$. Journal of Yangtze University (Nat Sci Edit), 2009, 6(3): 5-6(in Chinese).

[24] Chi D N, Hofmann W. Model-based loss minimization control of interior permanent magnet synchronous motors[A]. IEEE International Conference on Industrial Technology[C], Seville, Spain, 2015.

[25] 王莉娜, 朱鸿悦, 杨宗军. 永磁同步电动机调速系统PI控 制器参数整定方法 [J]. 电工技术学报, 2014, 29(5): 104-117.

[26] Wang Lina, Zhu Hongyue, Yang Zongjun. Tuning Method for PI Controllers of PMSM Driving System[J]. Transactions of China Electrotechnical Society, 2014, 29(5): 104-117(in Chinese).

[27] 李孟秋, 王龙. 一种改进的永磁同步电机低速无位置传感 器控制策略[J]. 电工技术学报, 2018，33(9)：1965-1973.

[28] Li Mengqiu, Wang Long. An improved low speed sensorless control strategy for permanent magnet synchronous motor[J]. Transactions of China Electrotechnical Society, 2018, 33(9): 1965-1973(in Chinese).

[29] Maren K, Kai S. Energy-efficient dynamic drive control for wind power conversion with PMSG : modeling and application of transfer function analysis[J]. IEEE Journal of Emerging and Selected Topics in Power Electronics, 2014, 2(1): $35-46$. 